\title{
ESTROGEN RECEPTOR POSITIVE SECRETORY BREAST CARCINOMA IN A BANGLADESHI ELDERLY WOMAN DIAGNOSED BY CORE NEEDLE BIOPSY
}

\author{
ABEDIN $\mathrm{N}^{1}$, BUSHRAH KN ${ }^{2}$, MUKTADIR MRM $^{3}$, DEY BP 4
}

\begin{abstract}
:
Secretory carcinoma is an extremely rare subtype of low grade invasive breast carcinoma and occurs infrequently in adults showing belligerent clinical course than in children. Surprisingly, it is the only epithelial carcinoma of breast representing characteristic balanced translocation. This paper reports a case of secretory breast carcinoma in an elderly post-menopausal woman in Bangladesh which is diagnosed by core needle biopsy. The majority of the case reports claimed this tumor to be a triple negative breast carcinoma, whereas our case report reveals a slightly different status in immunohistochemical context. A 78-years old, post-menopausal female presented with the chief complaints of painless, slowly enlarging lump in her left breast over nine months. Breast examination revealed a $2 x 1.5 \mathrm{~cm}$, well-delineated, firm, non-tender and mobile mass in the lower-outer quadrant of the left breast. Microscopically, the tumor was composed of cells containing PAS positive, diastase resistant and mucicarmine positive intra and extra-cellular secretory material and Alcian blue positive secretion was found extracellularly. The tumor cells showed strongly positive immunohistochemical reactivity for cytokeratin, EMA, $E$-cadherin and vimentin and focal positive reaction for $S-100$ protein and estrogen receptor but negative for both progesterone receptor and human epidermal growth factor receptor. Ki-67 proliferative index was about 4\%. Hence, the diagnosis of secretory breast carcinoma (SBC) was made. The patient underwent modified radical mastectomy with axillary clearance without any axillary lymphnode involvement in an external center. The patient has not received any radio or chemotherapy. The patient was under continuous follow-up for 3-months. Unfortunately, owing to the nation-wide lockdown for dreadful COVID-19 pandemic, the patient couldn't arrive at the hospital for further follow-up evaluation. Immunohistochemical (IHC) analysis has been proven to be an alternative potential method as gene analysis. Immunoprofiling of secretory breast carcinomas may play the pivotal role for selecting therapeutic strategies and predicting prognostic outcome of the disease process. Comprehending the immunohistochemical expression would make scope for applying targeted therapy in SBC patients in future.
\end{abstract}

Key words: Estrogen receptor positive, Secretory breast carcinoma

J Dhaka Med Coll. 2019; 28(2) : 216-223

\section{Introduction:}

In clinical practice, secretory breast carcinoma (SBC) is one of the infrequent subtypes (comprises of less than $0.15 \%$ ) of invasivebreast malignancies. At first, it was named as "Juvenile breast carcinoma" byMcDivitt and Stewart in $1966^{1}$ because of its intensive occurrence in children . However, in a review, Tavassoli and

1. Dr. Nafisa Abedin, Resident, Department of Pathology, Bangabandhu Sheikh Mujib Medical University, Dhaka, Bangladesh.

2. Dr. Kazi Nabila Bushrah, ShaheedSuhrawardy Medical College, Dhaka, Bangladesh.

3. Dr. Muhit Reza Md. Muktadir, Masters of Public Health, Medical Officer, National Tuberculosis Program, RDRS, Bangladesh.

4. Dr Bishnu Pada Dey, MD(Pathology), Assistant Professor, Department of Pathology, Bangabandhu Sheikh Mujib Medical University, Bangladesh.

Correspondence: Nafisa Abedin, Resident, Department of Pathology, Bangabandhu Sheikh Mujib Medical University, Dhaka, Bangladesh. Email:nafia.abedin@gmail.com (Orcid id: https://orcid.org/0000-0001-6682-7842)

Received: 04-07-2020

Revision: 16-08-2020

Accepted: 22-09-2020 
Norris stated that this carcinoma might occur over a broad range of age. Henceforth, it was renamed! ${ }^{2,3}$. Clinically, SBC follows an indolent course with better outcomes in adolescents whereas, in elderly age groups, clinical course is quite similar to that of $\mathrm{IDC}^{4}$. Cases of SBC have been identified in both sexes and female to male ratio is $6: 1^{3}$. The tumor can metastasize to lymphnodes and shows more aggressive course in male patients. As it has been proven that SBC can recur after local excision, therefore, total or modified radical mastectomy with axillary lymph nodes clearance is advocated as the standard therapeutic approach. Usually, the tumor is not chemosensitive, so treatment of the patients with single or combined chemotherapeutic agents showed no success. According to the opinion of Tavassoli and Norris, this tumor has a number of prognostic factors, for example - less than $2 \mathrm{~cm}$ of tumor size, less than 20 years of age at the time of diagnosis and well delineated tumor $\operatorname{margin}^{5}$.

\section{Case presentation:}

A 78-years old, post-menopausal female hailing from the district Chapainababganj, Bangladesh observed a painless, gradually growing mass in her left breast over nine months. She was diabetic and hypertensive for 25 years and had no previous or family history of breast cancer. At first, she took local traditional herbal medicine and homeopathic treatment. As with no improvement, she underwent evaluation in a medical center in her locality. Local examination of left breast revealed a $2 \times 1.5 \mathrm{~cm}$, well-demarcated,firm, non-tender and mobile lump in the lower-outer quadrant of left breast with no nipple discharge or nipple retraction. The lump was neither fixed to the underlying structure nor with the overlying skin. Ipsilateral axillary and supraclavicular lymph nodes were impalpable and right breast revealed unremarkable finding.USG of left breast showed that a hypoechoic solid mass $(2.2 \times 2.2 \mathrm{~cm})$ with irregular outline in lower quadrant of left breast. FNAC of the left breast lump of the patient revealed undifferentiated, atypical ductal cells with sheets like arrangement that seemed to be consistent with ductal carcinoma. Subsequently, the patient was advised for core needle biopsy and on March 19, 2020 in department of pathology, Bangabandhu Sheikh Mujib Medical University(BSMMU) we received three (03) formalin fixed breast core biopsy specimens. Grossly, the cores were gray-white and measured in between 1.5 and $0.4 \mathrm{~cm}$. Histopathology of paraffin embedded sections revealed an invasive carcinoma composed of tumor cells arranged in microcystic pattern with occasional micro papillary structures. The tumor cells also showed vacuolated, foamy cytoplasm with abundant intra and extra cellular pink secretions (PAS positive, diastase resistant, mucicarmine positive and Alcian blue positive only extracellular secretions) and bland uniform nuclei. Lympho-vascular and perineural invasions were indeterminate and mitosis was infrequent.

The tumor cells showed the following immunohistochemical features:

\begin{tabular}{lll}
\hline \multicolumn{2}{l}{ IHC findings of our case } & \\
\hline Strongly positive & Non-reactive & Variable expression \\
\hline EMA & Progesterone receptor (PR) & Estrogen receptor (ER) \\
& & {$[3.3 \%$ positive cells $]$} \\
Cytokeratin & HER2 & S100 (Focal expression) \\
Vimentin & & \\
E-cadherin & & \\
\hline
\end{tabular}



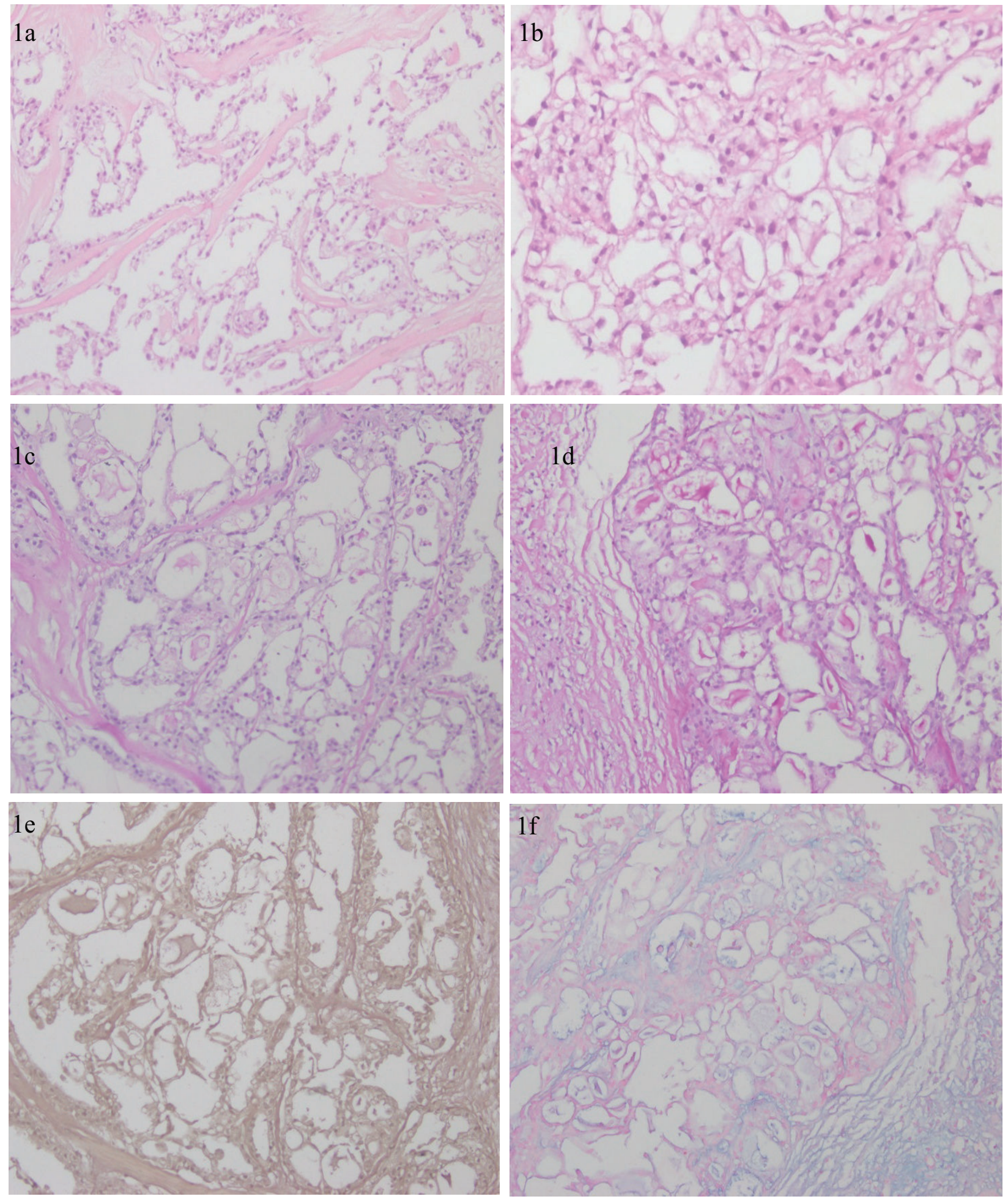

Fig.-1: $1 a(H / E$ 100X view), $1 b(H / E$ 200X view): The tumor shows microcystic and focally solid pattern. Tumor cells reveal vacuolated, foamy cytoplasm with ample intra and extracellular pink secretions and bland nuclei. 1c(PAS stained section,200X), 1d(PAS-D stained section,200X view):Abundant PAS Positive intra and extracellular material is present, which resists diastase digestion. The proteinaceous material is stained with mucicarmine(1e,200X view), and alcian blue positive secretion is present extracellularly(1f,200X view). 

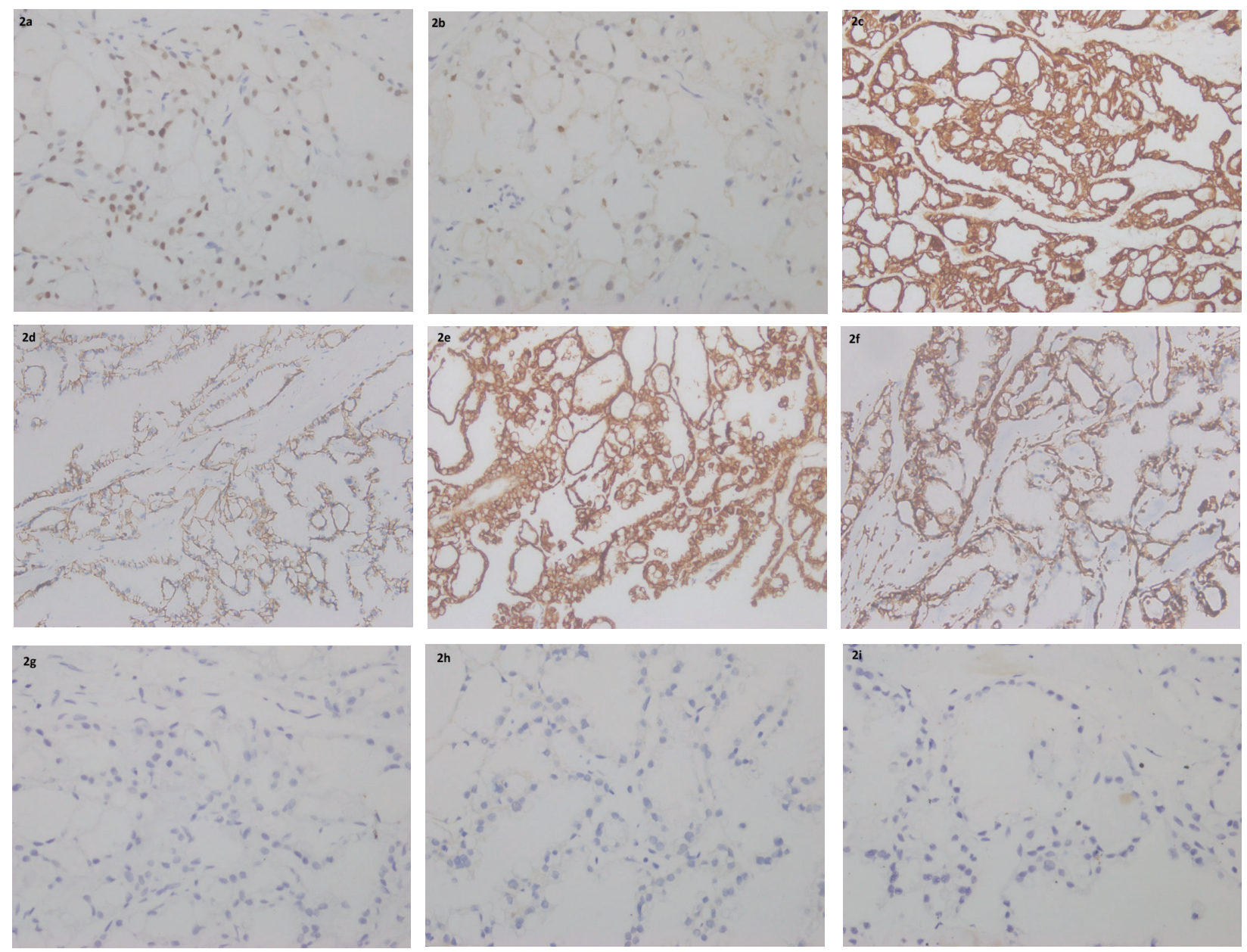

Fig.-2: The tumor cells showed focal positive reaction for estrogen receptor (2a,400X),S-100 protein(2b,400X), and, strongly positive immunohistochemical reactivity for cytokeratin (2c,200X), $E$-cadherin(2e,200X),EMA(2e,200X) and vimentin(2f,200X) and negative immune reactivity for both progesterone receptor $(2 \mathrm{~g}, 400 \mathrm{X})$, human epidermal growth factor receptor(2h,400X), and ki-67 proliferative index about $4 \%(2 i, 400 X)$

Hence, the diagnosis of SBC was confirmed. Although for definitive diagnosis, detection of ETV6-NTRK3 fusion protein is required, we could not detect mutation as a result of limited logistic support. Later on, she underwent modified radical mastectomy with axillary clearance in an external medical center and her clinical recovery was unmentionable. Unfortunately, the patient was lost during 3months of follow-up when national lockdownstarted for crucial situation of COVID19 pandemic.

\section{Discussion:}

SBC is one of the infrequently encountered breast carcinoma in clinical practice. Bottaet. $\mathrm{al}^{6}$ observed only one case of SBC among 3000 cases of breast malignancy and in a pathologic review of 10000 cases of breast carcinoma $\mathrm{Li}$ et,al ${ }^{7}$ found 15 cases of SBC. SBC occurs in both females and maleswith a wide range of age variation. Although it may develop in women age rangingfrom 3 to 83 years, inmost of the reported cases young females were more affected,with a median age is less than 30 years $^{8}$. AidaAyadi-kaddouret, $\mathrm{al}^{9}$ recorded SBC in a 66-years-old post-menopausal woman. Our patient is one of the elderly SBC cases reported in Bangladesh.

Patients typically present with slowly growing, painless, well-defined, mobile and readily palpable(occasionally non-palpable) breast lump. Alike invasive ductal carcinoma, not 
otherwise specified, the upper-outer quadrant of breast seems to be the most common site of involvementbySBCs. Nevertheless, it can develop in any of the breast quadrants ${ }^{8}$. Metastasis to axillary lymph node is rare, particularly if size of the tumors is smaller than $2 \mathrm{~cm}$. However, if the tumor metastasizes to lymph nodes, hardly ever, it can involve more than 3nodes! ${ }^{10}$. No case of hormonal abnormality in SBC has been reported yet. Additionally, in SBC no association of either family history or any endocrinopathy has been documented.

Since this patient informed that the lump was initially un-noticeable and gradually increasing in size over ten months, it can be comprehended that it had a slowly progressing behavior with an indolent course. As per the decision of the members of the European group for breast cancer screening (EGBCS, founded in 1982) application of ultrasound plays a significant role in breast cancer diagnosisspecially for unrecognizable lumps ${ }^{11}$. However, a number of different features can berevealed with the help of sonographic examination. In a case series for evaluating radiological features of SBC,Sung HeeMunet $\mathrm{al}^{12}$ revealed that among six secretory carcinomas, five were round or oval and one was tubular in shape; two out of six SBCs were relatively well-defined and four SBCs showed partially microlobulated edges in ultrasonography. The study also revealed hypoechoic and isoechoic internal echo texture by four and two cases respectively ${ }^{12}$. The sluggish growth pattern of the tumor and variable imaging findings make the diagnosis more challenging. However, on ultrasonography, the current case revealed $2.2 \times 2.2 \mathrm{~cm}$ hypoechoic solid mass with uneven outline locating in lower quadrant of left breast.

Cytologically, SBCs can be distinguished from other invasive breast carcinomas by the presence of abundant intra and extra-cellular secretory materials, granular eosinophilic cytoplasm with or without intracytoplasmic vacuolation of the cells and occasional presence of signet-ring cells. However, intracytoplasmic large vacuoles with copious proteinaceous material are the most characteristic feature of SBC. The monotonous malignant cells are arranged in tight, cohesive sheets as well as loose clusters. The cells show round nuclei with homogenously distributed chromatin. The background of the smear may exhibit abundant proteinaceous material ${ }^{13}$.

Both Core Needle Biopsy (CNB) and excisional biopsy have paramount importance for evaluation of architectural features and diagnosis of SBC. Morphologically, SBC can be characterized by a low-grade malignant tumor of epithelial origin with a wide range of structural variations, for instance, solid, microcysticor honeycomb and tubular arrangement. The tumor shows ample milk like secretion (Periodic Acid Schiff positive, diastase resistant and mucicarmine positive) both intracellularly and extracellularly and Alcian blue positive proteinaceous material may be present extracellularly ${ }^{14,3,15}$. Moreover, in CNB, SBC may show minute and focal islet like pattern of invasion and cribriform like glandular structure that can easily be confused with insitu carcinomas ${ }^{16}$.

Immunoprofiling of invasive breast carcinomas plays the pivotal role for determining the precise diagnosis, treatment strategies and overall prognosis of the disease process.The ETV6NTRK3 fusion gene ofSBCs are strongly associated with the positive expression of basal markers (for example: CK5/6, 14, 17, EGFR, vimentin etc.) ${ }^{17}$. In addition to that, immunohistochemically, SBC shows triple negative expression of ER, PR and Her2/neu receptors. Besides, few other recent studies have reported positive expressions of S100, Ecadherin, lactalbumin, EMA, mammoglobulin, polyclonal carcinoembryonic antigen (CEA) in SBC. Furthermore, markers like gross cystic disease fluid protein (GCDFP-15/BRST-2), monoclonal CEA etc. showed negative reactivity. Ki67 Proliferation index, shows variable activity starting from 1 to $34 \%^{8}$. Nevertheless, few examples of exceptional cases exhibiting positive expression of ER within the in-situ component of SBC have also been narrated in some recent case studies ${ }^{18,19}$. Our current case is one of the exceptional onesthat express positive ER markers. However, we could not evaluate EGFR,lactalbumin, mammoglobulin, polyclonal CEA, monoclonal CEA, GCDFP-15/BRST-2 IHC markers as a result of unavailability of these markers in our laboratory. 
The differential diagnoses of SBC include a broad range of both benign and malignant conditions.

\section{Differential Diagnoses of SBCs}

\begin{tabular}{ll}
\hline Malignant lesions(myoepithelial layer absent) & Benign lesions(myoepithelial layer present) \\
Invasive ductal carcinoma (IDC) & Juvenile papillomatosis with apocrine metaplasia \\
Cystic hypersecretory carcinoma (CHC) & Lactating adenoma \\
Glycogen rich carcinoma & \\
Mucinous carcinoma & \\
Tubular carcinoma & \\
Apocrine carcinoma & \\
Acinic cell carcinoma (ACCA) & \\
\hline
\end{tabular}

Histologically, SBC can be differentiated from benign lesions by absent myoepithelial cell layer ${ }^{3,14}$. HirokawaMet,al. recommended that ACCA of salivary gland and SBC are same histological entity because of their similar clinicpathological and immunohistochemical features ${ }^{20}$. However, Reis-Filhoet. al. opined that SBC and ACCA are separate entities by demonstrating through fluorescent in-situ hybridization (FISH) that ACCA did not harbor ETV6 genetic rearrangement detected in $\mathrm{SBC}^{21}$. CHC is characterized by striking secretory activity of tumor cells as well as markedly dilated ducts and cysts analogous to the thyroid follicles. The majority of cases of $\mathrm{CHC}$ are intraductal and shows positive immune reactivity for S-100 and alpha lactalbumin. The prognostic outcome of patients with SBC and its mimics varies depending upon biological characteristics of cancer and its spread at the time of diagnosis. However, the prognosis of SBC and ACCA is excellent unlike that of invasive $\mathrm{CHC}$ and $\mathrm{IDC}^{14}$. Hence, differentiation of SBC and its histomorphological mimics from each other as well as from other commonly encountered breast cancers is extremely important in clinical practice.

Interestingly, SBC is the unique epithelial tumor of breast harboring the characteristic balanced translocation $\mathrm{t}(12 ; 15)$ which creates a chimeric tyrosine kinase by fused gene ETV6(TEL)NTRK3.This balanced translocation known to be associated with two histologically identical childhood mesenchymal tumors namely infantile fibrosarcoma and cellular mesoblasticnephroma.Additionally, ETV6NTRK3 rearrangement is believed to be the primary molecular event in this subcategory of breast carcinoma as evidenced by presence of a constitutively active transcript in carcinoma cells and affirmation of the transforming ability of ETV6-NTRK3 of normal murine mammary epithelial cells. The fused ETV6-NTRK3initiates two major pro-growth promoting pathways for proliferation and survival of the transformed cells, including RAS-MAPK(activation of transcription) and PI3K-AKT (pro-growth metabolism) signals ${ }^{17}$. However, Cristina Tognon et,al.2002 confirmed expression of ETV6-NTRK3 in 92\%(12 of 13) of SBC and demonstrated that this specified genetic lesion belongs to the phenotypic triple negative and basal like varieties of breast carcinomas ${ }^{22}$. Unfortunately, owing toscarce needful resources (polymerase chain reaction or fluorescent in situ hybridization), we could not evaluate the aforementioned genetic mutation.

Surgical approach has been regarded as the cornerstone of management of SBCs. However, due to limited number of case reports, no specific and published guidelines of management are available yet. One of the main purposes of modified radical or radical mastectomy for the patients harboring the disease is to avoid delayed local recurrence ${ }^{23}$. However, in younger patients where breast has not fully developed, lumpectomy followed by late reconstruction is considered as lucrative. In general, the incidence of axillary lymphnode metastasis is approximately $30 \%$ in both male and female regardless of age. Hence, axillary clearance is recommended by some authors, if size of tumor is $\leq 2 \mathrm{~cm}^{24}$. 
Efforts onadjuvant radiation and chemotherapy have been attempted for the remedial treatment of the disease without favorable outcome. This observation falls contrary to the excessive chemosensitivity exhibited by childhood mesenchymal tumor, namely, infantile fibrosarcoma and mesoblasticnephroma to conventional chemotherapeutic agents. Currently, radiation and chemotherapy are considered mostly for the rare presentation of advanced stage of the disease. The identification of the distinctive ETV6-NTRK3 balanced translocation may pave way fordesigning drugs used in personalized medical therapy for this carcinoma.

\section{Conclusion:}

Being an exceptionally uncommon neoplasm, incidence of SBC is more infrequent in elderly women. Recent findings demonstrate that distinctive immunohistochemical and genetic attributes can distinguish SBC from frequently encountered ducal carcinoma of the breast. As major proportion of SBC donot exhibit ER, the success of hormone therapy has not been evaluated yet. Our study is reporting an unusual case in which ER show positive expression.Nevertheless, few cases of ER positive SBC have been reported, compatible with the features denominated in the current study. Advanced technologies, such as gene expression profiling (GEP), have evidently enabled us to gather elaborate knowledge in an effort to establish the diagnosis of SBC more effectively. However, economical restrains and impractical application of GEP have led us to look out for another potential surrogate. Immunohistochemical (IHC) analysis has been proven to be a dynamic method as an alternative to GEP. Immunoprofiling of invasive breast carcinomas plays the pivotal role for determining the treatment strategies and overall prognosis of the disease process. Therefore, immunohistochemical analysis prior to initiating treatment for secretory breast carcinoma should be brought under routine practice. Better understanding of immunohistochemical expression would intuit in applying personalized medicine in SBC patients in future.

\section{References:}

1. F.A.Tavassoli HJN. Secretory carcinoma of the breast. Breast J. 1980;45:2404-13.

2. Horowitz DP, Sharma CS, Connolly E, Gidea-Addeo D, Deutsch I. Secretory carcinoma of the breast: Results from the survival, epidemiology and end results database. Breast [Internet]. 2012;21(3):3503. Available from: http://dx.doi.org/10.1016/ j.breast.2012.02.013

3. Sharma V, Anuragi G, Singh S, Patel P, Jindal A, Sharma RG. Secretory Carcinoma of the Breast: Report of Two Cases and Review of the Literature. Case Rep Oncol Med. 2015;2015(Figure 1):5-10.

4. Anderson P, Albarracin CT, Resetkova E. A large, fungating breast mass. Secretory carcinoma with apocrine differentiation. Arch Pathol Lab Med. 2006;130(4):50-2.

5. Gabal S, Talaat S. Secretory Carcinoma of Male Breast: Case Report and Review of the Literature. Int J Breast Cancer. 2011;2011:1-5.

6. Botta G, Fessia L, Ghiringhello B. Juvenile milk protein secreting carcinoma. Virchows Arch A Pathol Anat Histol. 1982;395(2):145-52.

7. Li D, Xiao X, Yang W, Shui R, Tu X, Lu H, et al. Secretory breast carcinoma: A clinicopathological and immunophenotypic study of 15 cases with a review of the literature. Mod Pathol. 2012;25(4):567-75.

8. Vasudev P, Onuma K. Secretory breast carcinoma: Unique, triple-negative carcinoma with a favorable prognosis and characteristic molecular expression. Arch Pathol Lab Med. 2011;135(12):1606-10.

9. Kaddour AA. Secretory Carcinoma of the Breast in an Elderly Woman. J Cytol Histol. 2015;6(3):6-8.

10. V S, Aisha A, Ravinder T. Secretory carcinoma of the breast. Breast J. 2006;11(1).

11. Teh W, Wilson ARM. The role of ultrasound in breast cancer screening. A consensus statement by the European Group for Breast Cancer Screening. Eur J Cancer. 1998;34(4):449-50.

12. Mun SH. Secretory carcinoma of the breast. Breast J. 2008;27(2):947-54.

13. Alenda C, Aranda FI, Seguí FJ, Laforga J. Secretory carcinoma of the male breast: Correlation of aspiration cytology and pathology. Diagn Cytopathol. 2005;32(1):47-50.

14. Osako T, Takeuchi K, Horii R, Iwase T, Akiyama F. Secretory carcinoma of the breast and its histopathological mimics: Value of markers for differential diagnosis. Histopathology. 2013; 63(4):509-19.

15. Yorozuya K, Takahashi E, Kousaka J, Mouri Y, Yoshida M, Fujii K, et al. A case of estrogen receptor 
positive secretory carcinoma in a 9-year-old girl with ETV6-NTRK3 fusion gene. Jpn J Clin Oncol. 2012;42(3):208-11.

16. Fatma Aktepe, Dauren Sarsenov VO. Secretory carcinoma of the breast. Breast J. 2016;12(2):1746.

17. Laé M, Fréneaux P, Sastre-Garau X, Chouchane O, Sigal-Zafrani B, Vincent-Salomon A. Secretory breast carcinomas with ETV6-NTRK3 fusion gene belong to the basal-like carcinoma spectrum. Mod Pathol. 2009;22(2):291-8.

18. Sato T, Iwasaki A, Iwama T, Kawai S, Nakagawa T, Sugihara K. A rare case of extensive ductal carcinoma in situ of the breast with secretory features. Rare Tumors [Internet]. 2012;4(4):169-71. Available from: https://doi.org/10.4081/rt.2012.e52

19. Yilmaz KB, Pak I, Atalay C, Özaslan C. Histopathological and clinical characteristics of secretory carcinoma of the breast. Turkish J Med Sci. 2009;39(1):155-9.

20. Hirokawa M, Sugihara K, Sai T, Monobe Y, Kudo H, Sano N, et al. Secretory carcinoma of the breast: A tumour analogous to salivary gland acinic cell carcinoma? Histopathology. 2002;40(3):223-9.

21. Reis-Filho JS, Natrajan R, Vatcheva R, Lambros MBK, Marchió C, Mahler-Araújo B, et al. Is acinic cell carcinoma a variant of secretory carcinoma? A FISH study using ETV6 "split apart" probes. Histopathology. 2008;52(7):840-6.

22. Tognon C, Huntsman D, Sorensen PHB. 26 Expression of the ETV6-NTRK3 gene fusion in human secretory breast carcinoma. Handb Immunohistochem Situ Hybrid Hum Carcinomas. 2002;2(November).

23. C Arce, D Cortes-padilla, DG Huntsman, MA Miller, A Duennas Gonzalez AA. Secretory carcinoma of the breast containig the ETV6-NTRK3 fusion gene in a male/ :case report and review of the literature [Internet]. Vol. 3, Tandlægebladet. 2005. Available from: http://www.wjso.com/content/3/1/35

24. Richard G, Hawk JC, Baker AS, Austin RM. Multicentric adult secretory breast carcinoma: DNA flow cytometric findings, prognostic features, and review of the world literature. J Surg Oncol. 1990;44(4):238-44. 